\title{
Advances in nanomedical applications: diagnostic, therapeutic, immunization, and vaccine production
}

\author{
Amr El-Sayed ${ }^{1} \cdot$ Mohamed Kamel $^{1}$
}

Received: 13 December 2018 / Accepted: 6 September 2019/Published online: 16 September 2019

(C) Springer-Verlag GmbH Germany, part of Springer Nature 2019

\begin{abstract}
In the last decades, nanotechnology-based tools started to draw the attention of research worldwide. They offer economic, rapid, effective, and highly specific solutions for most medical issues. As a result, the international demand of nanomaterials is expanding very rapidly. It was estimated that the market of nanomaterials was about $\$ 2.6$ trillion in 2015 . In medicine, various applications of nanotechnology proved their potential to revolutionize medical diagnosis, immunization, treatment, and even health care products. The loading substances can be coupled with a large set of nanoparticles (NPs) by many means: chemically (conjugation), physically (encapsulation), or via adsorption. The use of the suitable loading nanosubstance depends on the application purpose. They can be used to deliver various chemicals (drugs, chemotherapeutic agents, or imaging substances), or biological substances (antigens, antibodies, RNA, or DNA) through endocytosis. They can even be used to deliver light and heat to their target cells when needed. The present review provides a brief overview about the structure and shape of available NPs and discusses their applications in the medical sciences.
\end{abstract}

Keywords Cancer therapy $\cdot$ Imaging agents $\cdot$ Nanotechnology $\cdot$ Nanomedicine $\cdot$ Nanovaccines

\section{Introduction}

The development of new industrial tools which enabled the manipulation of the materials at their nanoscale provided the medical science with opportunities for evolutionary new applications. This, in turn, opened the door for new solutions for old medical problems such as drug-resistant pathogens, vaccine development, and cancer therapy (Manuja et al. 2012). Since 1974, the term (Nanotechnology) started to describe the techniques applied for the manipulation of the materials at their nanoscale making at least one of their three dimensions (3D) about 1-100 nm (Troncarelli et al. 2013). Other expressions were adapted to the new (Nano) situation, so that terms like Nanomaterials, Nanomedicine, Nanovaccines, or Nanotheranostics started to be introduced. The nanomedicine is the application of various nanotechnological tools to

Responsible editor: Philippe Garrigues

Mohamed Kamel

m_salah@staff.cu.edu.eg

1 Faculty of Veterinary Medicine, Department of Medicine and Infectious Diseases, Cairo University, Giza, Egypt develop better solutions for medical problems (Mohantya et al. 2014). On the other hand, the nanotheranostics are hybrid formulations which have diagnostic and therapeutic properties at the same time (Rizzo et al. 2013). Meanwhile, the nanomaterials are the manipulated materials to have one of their $3 \mathrm{D}$ in nanosize. It is worthy to mention that nanomaterials have different chemical, physical, biological, and therapeutic properties than their counterparts. The source of theses nanomaterials is natural or engineered or incidental as a byproduct of mechanical or industrial processes (Jeevanandam et al. 2018). The reduction in size makes them not only more reactive, soluble, and efficient but also may provide them with new properties which are not present in their counterpart (Swain et al. 2015; Troncarelli et al. 2013). A good example of such changes in material properties is the soybean oil which traditionally has little applications in medical fields. However, when converted to nanodrops (emulsification), it gains high potential to destroy multidrug-resistant pathogens (Hajipour et al. 2012; Hakemi-Vala et al. 2017). This potential is achieved through its physical properties caused by the surface tension. Upon coming in contact with bacterial/viral envelope, the drops merge with the membrane and kill the pathogens. This new category of antibiotics is safe as they do not harm the eukaryotic cells and effective as no 
bacterial resistance can be developed against it because its mode of action is physical not chemical (Chakravarthi and Balaji 2010).

Historically, NP development started with Paul Ehrlich and then initial trials by Ursula Scheffel and colleagues and the extensive work by the group of Professor Peter Speiser at the ETH Zurich in the late 1960s and early 1970s are delineated from a personal viewpoint (Kreuter 2007). Momentous attention is paid to the years from 1970 to the early 1980s. Further evolutions emerging from this work are also followed, and concentrate is put particularly strong advances such as nanoparticles for drug delivery crossing the blood-brain barrier (BBB) (Kreuter 2007). There are many types of NPs, the size, shape, and origin determine to a large extent the application of the nanoparticles (NPs). The most common types are listed in Table 1 and Fig. 1. The NPs can be used as vehicles to deliver their loaded substances through two mechanisms: (1) encapsulation using lipid-based capsules or polymer-based capsules and (2) polymer-based structures using natural polymers or synthetic polymers (Troncarelli et al. 2013). In general, the NPs have several advantages compared with conventional preparations in the field of diagnostics and therapeutics. They are biocompatible and mostly safer (Jurj et al. 2017). They can cross the BBB and other physiological barriers (Ceña and Játiva 2018; Zhou et al. 2018), and can kill intracellular and multiple drug-resistant pathogens efficiently (Talukdar et al. 2014). They provide new approaches for vaccine development and gene therapy (Awate et al. 2013; Riley and Vermerris 2017; Sridharan and Gogtay 2016). The present work discusses the medical applications of nano-products in diagnostics as well as in therapeutic field.

\section{Uses of nanotechnology in medicine}

The employment of nanotechnology in medicine empowered the increase of half-life time of the drugs by improving drug internalization, decreasing drug degradation/clearance, and through providing slow-release mechanisms of loaded drugs. The circulation time of the NPs can be controlled by changing the surface charge of the NPs according to the goal of their application. While the positive charge increases the internalization of the NPs, the negative charges extend the circulation time in the blood stream. In addition, they increase the bioavailability of the drugs, and improve the water solubility of hydrophobic preparations. They modify pharmacokinetics and exactly deliver the drugs where they are needed. Therefore, they decrease the side effects and reduce the required doses. They may be loaded with one or more drugs in parallel. New generation of nano-robotics can independently make decisions according to the situation and help the surgeons during the operations (Freitas Jr 2005; Freitas 2005; Jurj et al. 2017; Troncarelli et al. 2013). The nanotechnology utilization in medicine has been summarized in Fig. 2.

\section{Diagnostics}

Multipurpose nanosized sensors were designed to detect different pathological parameters, foreign proteins/antigens, and toxic substances. Recently, bio-barcodes were also developed to target protein disease markers as the PSA (prostate specific antigen). Such biosensors can diagnose prostatic cancer very early based on anti-PSA antibodies. The sensitivity of the developed assay utilizing bio-barcode for the diagnosis of prostatic cancer is about one million times more than the conventional methods used at the time (Manuja et al. 2012).

\section{As imaging agents}

Ideal imaging agents are now developed using various nanomaterials due to the unique optical, chemical, and physical properties. They provide better contrast, controlled biodistribution, and multi-model imaging for ultrasound, MRI, PET, and SPECT. Due to the leakage in capillaries built in the cancer tissues, the NPs tend to accumulate in the tumor interstitial spaces. This allows the diagnosis of very small lesions which cannot be detected by classical imaging agents (Chapman et al. 2013).

Although many classes of NPs can be used as imaging agent, the physical characters of the QD (such as their high photostability and resistance to photobleaching) made them ideal for use in imaging purposes. They can be utilized as fluorescent probes and highly sensitive low-cost biosensors. The degree of the fluorescent emission of the QD can be controlled according to the size of the QD used. They have varied additional advantages over the classical fluorescent dyes, being brighter, easy to visualize, and stay longer in the body (Meena et al. 2018). Multimodal imaging provides advanced imaging benefits such as the combination of paramagnetic QD for fluorescent imaging at the same time with MRI (Minchin and Martin 2010).

\section{For the diagnosis of cancer}

There are over 200 types of cancer; every type has its own characteristics. Some of them (e.g.. lung cancer) are difficult to diagnose in the early curable stages through conventional techniques where the cancer is still a localized slow-growing tumor (Hirsch et al. 2003) It is estimated that the cancer cells require about 10 years since their first appearance before they can be diagnosed by conventional methods. Late diagnosis of cancer usually complicates the case and worsens the prognosis especially if the cancer metastasized to distant sites such as the liver and brain (Martin et al. 2013). In addition, the traditional imaging agents are renal toxic and cannot be applied in 


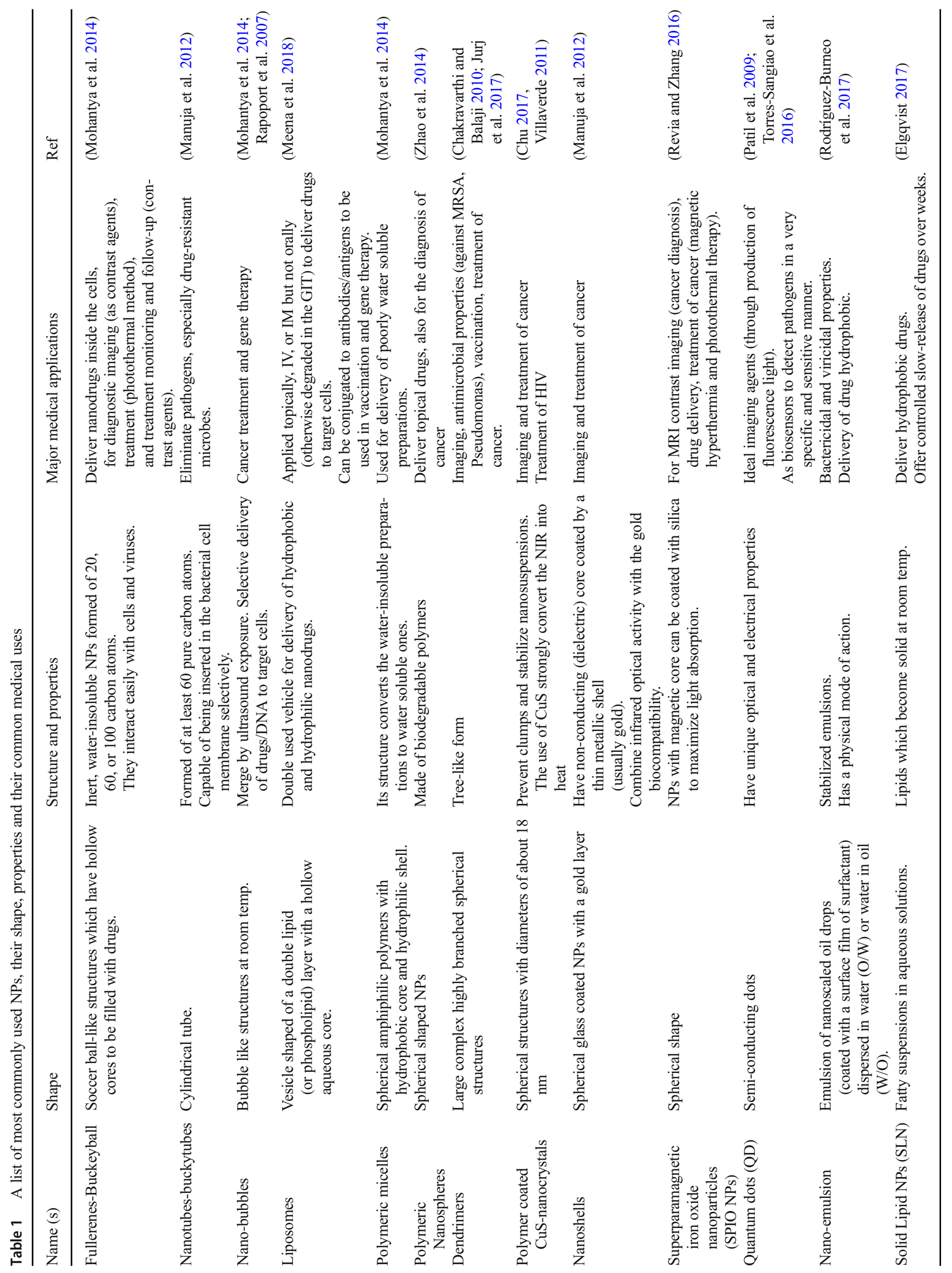


patients with renal problems. They are inaccurate in the diagnosis of lesions less than $1 \mathrm{~cm}$ (Krishnan and George 2014). Due to the optical, chemical, and physical characters of the nanoshells, they are favored for use as a scanner for skin cancer which can differentiate among various malignant and benign types. Tumor markers including certain proteins (e.g., epidermal growth factor receptor or the proepithelin) are characteristic for cancer cells and are usually widely distributed on their outer surface of the malignant cells. The development of nanosensor chips which carry special antibodies to these markers provides an early and sensitive diagnostic tool for cancer (Monami et al. 2009; Probst et al. 2013). The use of gold NPs as bio-barcode assay provides a very accurate diagnostic tool for prostatic cancer. Trials to develop an electrochemical immunosensors for the diagnosis of cancer are under development (Li et al. 2017; Remião et al. 2018).

\section{For the diagnosis of infectious diseases}

NPs allow rapid, accurate, and cost-effective diagnosis of many infectious diseases. Among these systems, the developed fluorescent silica NPs (FSNPs) can detect infections with mycobacterium tuberculosis complex (MTB), Salmonella typhimurium, or S. aureus within only 4 h (Qin et al. 2007; Shangguan et al. 2015; Wang and Kang 2016).

Liposome-based systems were also developed for rapid detection of food-borne toxins such as cholera or botulinum toxins. The test (sandwich fluoro-immunoassay) is carried out on an antibody-coated microtiter plate where the liposomes are labeled with fluorescent markers specific for target toxins (Ahn-Yoon et al. 2003; Ahn-Yoon et al. 2004).

Recently, new approaches based on colorimetric, fluorescence, mass spectrometry, and electrochemical assays, various metal NPs known as surface plasmon resonance (SPR) were adapted to detect DNA/RNA sequences, or proteins and even small analytes accompanying infections (Pedrosa and Baptista 2015).

Zhang et al. developed a single quantum dots-based nanosensor specific for the detection of HIV-1 and HIV-2 viruses (Zhang and $\mathrm{Hu}$ 2010). The assay requires a very low sample volume and delivers the results in a short time with a very high sensitivity level. Meanwhile, fluorescent immunosensors were also designed to diagnose Salmonella spp. even if present at a very low concentration. The technique depends on the use of simple cellulose-based swabs which allow the examination of a large number of samples in a short time (Tang et al. 2016). Similar assays are also applied for the diagnosis of other infectious diseases such as HVB, HCV, and Serratia marcescens infections (Ebrahim et al. 2015; Klostranec et al. 2007) Other HCV and HBV diagnostic assays using immune-gold silver staining with Au-NPs are under investigation. The pilot study results are promising (Duan 
Fig. 1 Schematic illustration displaying the most commonly used NPs in medicine

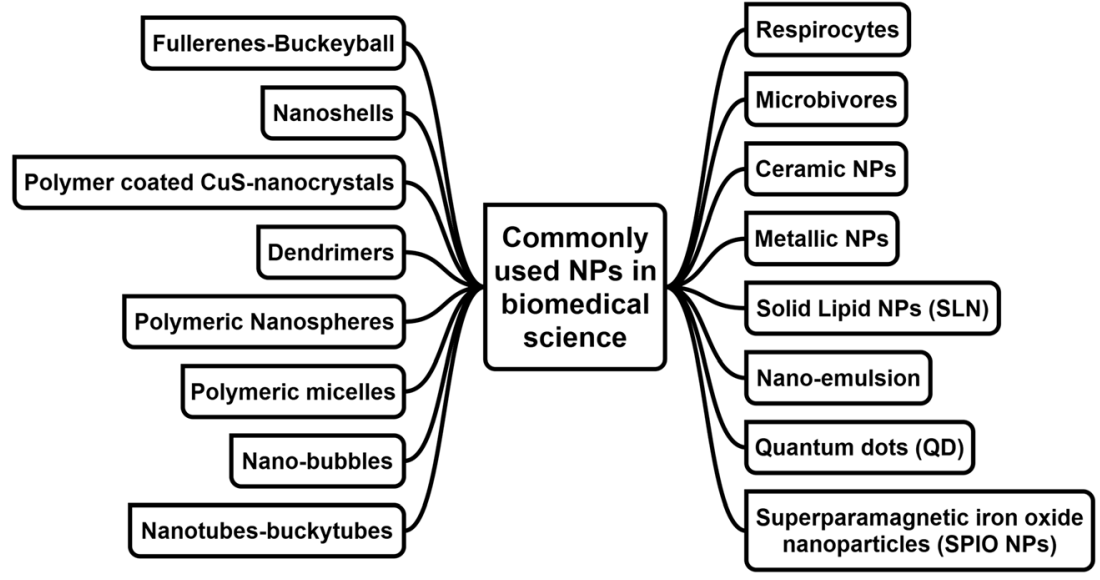

et al. 2005; Goluch et al. 2006; Pedrosa and Baptista 2015; Qasim et al. 2014).

NPs are also used for the early diagnosis of acute renal failure (ARF) and renal ischemia. Classical diagnostic assays for the diagnosis of ARF depend mostly on the measurement of serum creatinine level. In opposite to them, the newly developed dendrimer-encapsulated contrast nanoparticle-based magnetic resonance imaging (MRI) assay offers early diagnosis even before serum creatinine level gets elevated which saves time and allows early interference (Dear et al. 2006).

\section{Therapeutic applications}

The evolutionary applications of NPs in medicine opened the door for the treatment of many diseases which were recognized to be fatal or have bad prognosis records. They are used not only to treat infectious diseases but also non-infectious diseases such as metabolic/hormonal disorders, autoimmune diseases, cancer, and inflammations. They can kill multidrugresistant pathogens, cross the $\mathrm{BBB}$, and can even supply the body with oxygen and functionally replace RBCs/WBCs (Krishnan and George 2014).

\section{For the treatment of infectious and non-infectious diseases}

The development of smart drugs which become active only in their target organs under specific circumstances offered flexibility, specificity, and individuality in the treatment of every case separately. Smart drugs also control the required doses providing a slow-release mechanism over days or even weeks. The application of these new therapeutic approaches enabled efficient elimination of viruses and intracellular organisms. Nanosized streptomycin and doxycycline showed greater potential in the treatment of Brucella melitensis infection than their counterparts (El-Sayed 2018; Manuja et al. 2012; Troncarelli et al. 2013).

Examples for some therapeutic preparations which are under development include the use of the nanoformulations (1) PEG-AmB-LIP and PLGA loaded with Amphotericin B for the treatment of leishmaniasis and even systemic mycotic infections. The administration of the drug as a nanoparticle
Fig. 2 The different applications of nanoparticles in medical science: diagnostic, therapeutics, and immunization

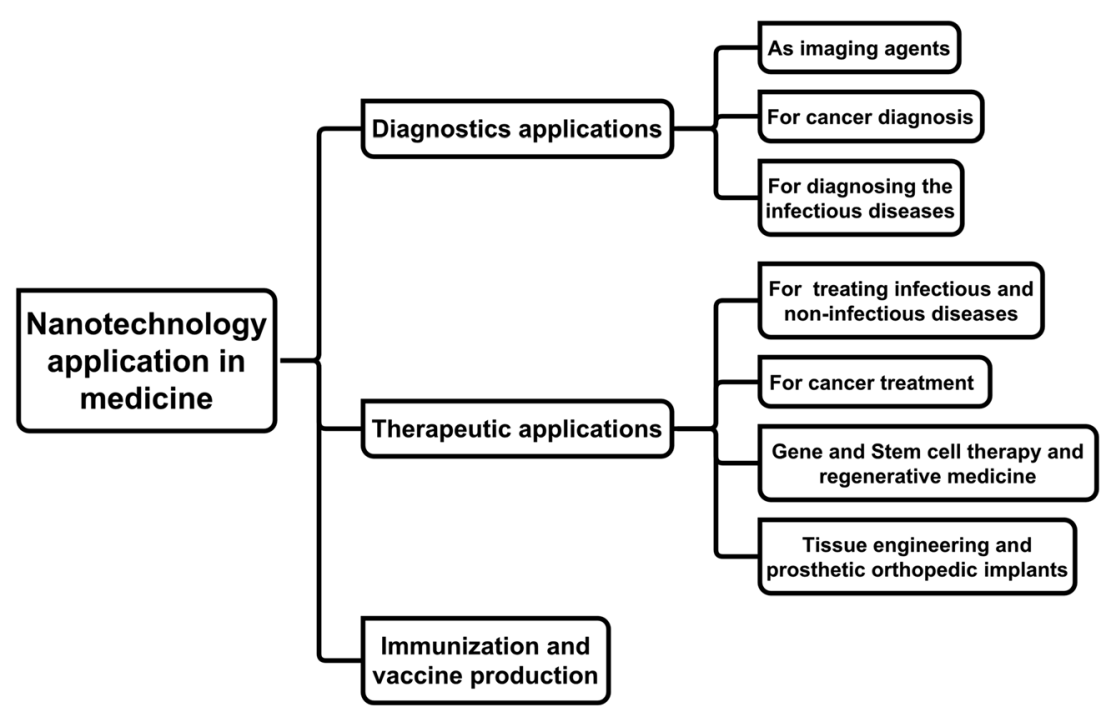


increases the drug efficiency for the treatment of the intracellular Leishmania infections by 20 -folds compared with the same active ingredient when given in the conventional form. (2) Isobutyl cyanoacrylat loaded with ampicillin for the treatment of salmonellosis increases the therapeutic efficiency of ampicillin to eliminate salmonellosis by 120 -folds (Manuja et al. 2012). (3) PEO-B-PAA_1Na loaded with streptomycin and doxycycline revealed promising results in the elimination of brucellosis from the liver and spleen in mice (Seleem et al. 2009). (4) The use of PLGA containing rifampin, isoniazid, and pyrazinamide completely eradicated mycobacterium tuberculosis from lab animals suffering from generalized TB infection (Sharma et al. 2004).(5) Liposomes loaded with etanidazole could treat trypanosoma cruzi infections in lab animals (Morilla et al. 2005).

Acquired antibiotic resistance represents an international public health hazard and the multi-drug-resistant pathogens such as the XDR-/ TDR-/ MDR-TB, MRSA, and enterobacteria became a serious concern. NPs offer new chemical and physical solutions for the elimination of antibioticresistant pathogens. The NPs can perform their mission selectively and at the same time with minimal side effects/host toxicity. Additionally, they can reduce the bacterial attachment and biofilm formation (Torres-Sangiao et al. 2016).

The recently developed silver ring-coated superparamagnetic iron oxide NPs overcomes the known limitations of using silver NPs in pathogen control. The new NPs are biocompatible, have a clear antimicrobial effect, and are less toxic to host cells. They penetrate deeper into the biofilm to eliminate the pathogens and are easily directed by external magnetic fields (Gupta et al. 2016; Mahmoudi and Serpooshan 2012).

While trials to use iron chelators for the treatment of drugresistant TB isolates showed promising results, it was found that nitric oxide (NO)-loaded nanoparticles are effective in the elimination of MRSA. The NO-NPs have also an antiinflammatory effect and allows rapid wound healing with minimal scar tissue formation. Meanwhile, the gold NP dispersed on zeolites provided a radical and rapid solution for antibiotic-resistant E. coli and Salmonella typhi strains (Talukdar et al. 2014; Troncarelli et al. 2013). Similarly, infections with $S$. aureus could be successfully eliminated by the administration of antimicrobial peptides anchored to goldcoated NPs. The NPs were directed to the bone marrowderived mesenchymal stem cells of the patients (Riley and Vermerris 2017).

Both $\mathrm{ZnO}$ and $\mathrm{CuO}$ were also proven to be efficient in the treatment of MDR pathogens. They kill the bacteria by the induction of membrane disruption and generation of reactive oxygen species (ROS). They were successfully applied in the treatment of MSSA, MRSA, MDR, E. coli, Strept. mutans, and Klebsiella pneumoniae (Beyth et al. 2015; Dastjerdi and Montazer 2010; Hameed et al. 2016). The use of nanoemulsions was proven to be efficient as antimicrobial agent.
The oil drops merge with the pathogen membrane and cause its death in a physical way (Chakravarthi and Balaji 2010).

In a parallel line, the NPs can also aid in the treatment of many non-infectious chronic/metabolic diseases. The administration of encapsulated secretory cells offers new aspects for hormonal and enzymatic treatment in deficiency disease. For this purpose, NPs containing nanopores are used for supplying the body with deficient enzymes/hormones by keeping the secretory cells in the internal chambers of the particles, and the size of the pores allow the passage of the secreted materials but not the secretory cells themselves. Rat pancreatic beta cells which were inoculated in NPs remained viable and secrete insulin for several weeks as they were protected from the attacks of the immune system. This approach is promising in the treatment of type 2 diabetes (Leoni and Desai 2004). Another approach for the treatment of diabetes was tested. The concept depends on the co-loading of an electro-active hydrogel membrane with insulin and glucose oxidase enzyme. The membrane acts as a biosensor, in the presence of high concentration of glucose, and the membrane releases insulin in proportions equivalent to the concentrations of glucose in the medium (Brahim et al. 2002).

The NPs could be applied for diminishing the damaging effects of ischemia. In the body, the superoxide dismutase (SOD) is the major "antioxidant defense system" against free superoxide anion radicals. Trials to convey SOD-loaded NPs to the injured sites due to anion free radical in laboratory animals succeeded to minimize tissue damage and fibrosis. The application of this tool in the treatment of renal ischemia or stroke reduces the size of damaged tissues by $50 \%$ (Brede and Labhasetwar 2013). Other chronic diseases could be treated with the aid of nanotechnology such as Parkinson's disease (Ganesan et al. 2015) and psoriasis (Gupta et al. 2016).

\section{For the treatment of cancer}

In the last years, surge reports about resistance of cancer cells to treatment with conventional approaches were published. Malignant cells may develop drug resistance through different mechanisms including (1) increasing of drug metabolism, (2) mutation of drug target receptors, (3) increase drug efflux pump, (4) changes in tumor microenvironment to tolerate hypoxia and enhances tumor growth and metastasis, (5) quiescent and stem cells which resist apoptosis and survive the treatment, and (6) higher DNA repair capacity to prevent cell apoptosis (Casals et al. 2017). These factors may be either intrinsic or acquired mechanisms. The intrinsic resistance is triggered due to initial tumor features before starting the therapy which makes the chosen drug ineffective to eliminate this cancer type, while the acquired resistance is developed later on after the beginning of the therapy due to selective pressure. In such cases, the tumor mass is clearly reduced and apparent healing occurs although some clones escape the healing, 
develop resistance, and start to expand and metastasis. A common example is the metastatic castration-resistant prostate cancer (mCRPC) (Casals et al. 2017; Livney and Assaraf 2013).

Conventional chemotherapeutic approach for cancer treatment aims mainly to delay cancer progress and metastasis by inhibiting physiological cell functioning through the interference with (1) the replication and repair of DNA, (2) protein synthesis, and (3) cell division mechanisms. However, the use of chemotherapy is not exclusive for cancer cells alone. In comparison with healthy tissues, cancer cells have higher metabolic and glycolytic activities which make them more susceptible to irradiation and DNA damage. However, it is a challenge to protect surrounding tissues from being exposed to irradiation to prevent their damage without reducing the therapeutic doses given to the cancer cells. Therefore, the development of new approaches was necessary to enable sensitive identification, selection, targeting, and elimination of cancer cells (Aparicio et al. 2013).

An ideal cancer therapy should have certain properties: being able to completely eliminate the malignant cells, safe for use, has minimal side effects, and is cost-effective (Casals et al. 2017). None of the available conventional approaches can provide these properties. Among the obstacles facing the conventional assays are (1) malignant cell resistance to cell division inhibitors and chemotherapy (Jurj et al. 2017). (2) The application of chemotherapy is also problematic as it is substantial to give high doses in order to reach the therapeutic dose in the tumor tissue. Therefore, the healthy tissues suffer as well from the high doses administered. As a result, it is critical to balance between giving the correct dose or giving low (sub-therapeutic) interrupted to help recovery of the body. It is also important to administrate high doses of the drugs as the therapeutic agents do not target the tumor tissues and get distributed all over the body and are mostly cleared by the macrophages to the reticuloendothelial system (Blanco et al. 2011). The accumulation of enough amounts of the therapeutic agent in the tumor can also be made more difficult when necrotic lesions with poor blood flow are present (Cao et al. 2011). (3) Cancer therapy may also fail due to the high survival power of the cancer stem cells (CSC) which are characterized by their high DNA repair capability, overexpression of anti-apoptotic factors, high hypoxic stability, presence of detoxifying enzymes, and effective drug efflux transporters. The survival CSC after chemo/radiotherapy will lead to recurrence of the tumor and its spread to give metastasis in other organs (Vinogradov and Wei 2012).

The use of NPs in cancer therapy provides individualized therapeutic programs, i.e., every patient becomes a suitable therapeutic protocol according to the type/stage of the cancer in addition to his/her health condition. They specifically target the tumor, overcome drug resistance problems, minimize the given dose which reflects positively on the general health condition of the patients, enable combining more than one agent in the same formulation, follow and trap metastatic cells anywhere in the body, detect the tumor much earlier than conventional diagnostic methods, provide faster healing / recovery, decreases the risk of infection, reduce the cost of treatment, and improves the compliance (Elgqvist 2017; Krishnan and George 2014).

Therapeutic agents can be directed to the tumor cells through active targeting where tumor-specific antibodies are conjugated to the NPs. They target certain biological structures overexpressed in malignant tissues. Meanwhile, passively targeting can be performed through targeting certain cell functions or environments. Passive targeting depends on the enhancement of cell permeability and retention effect (EPR) exhibited by some cancer types. Malignant tissues are characterized by the formation of perforated leaky blood vessels and inefficient lymph drainage. The small-sized NPs pass through the pores in the walls of the leaky vessels into the tumor tissues and are retained there. Passive targeting, in opposite to active targeting, is restricted to some but not all cancer types (Jurj et al. 2017; Revia and Zhang 2016). After their arrival to their target, the therapeutic agents can enter the cancer cells by different mechanisms such as endocytosis. Following the internalization of the drugs into the cells, the therapeutic agents get trapped in the endosomes where they are enzymatically degraded by the lysozymes. Alternatively, the NPs may utilize another pathway to enter the cells such as via pore formation in the lipid bilayer of the membrane allowing the agents to enter the cells. The proton sponge effect ( $\mathrm{pH}$-buffering effect) mechanism helps also in the release of the entrapped drugs by allowing the inflow of water and ions into the endosomes leading to the rupture of the endosomal membrane (Varkouhi et al. 2011). The endosomal membrane may also be destabilized by fusogenic peptides or photochemically in which the endosomal membrane is ruptured by the released singlet oxygen following light exposure (Jurj et al. 2017).

Magnetic NPs are also used for the diagnosis (imaging agent) and the therapy (theranostic platform) of cancer. The produced alternating magnetic fields (AMF) can be applied for heating the tissues (Fortin et al. 2008). Among other factors, such as the source/form of iron used, NPs made of iron oxide with nano-crystalline magnetite $(\mathrm{Fe} 3 \mathrm{O} 4)$ cores are the most commonly used in cancer treatment due to their high biocompatibility and biodegradability. They are easily and cost-efficiently synthesized. Another influencing factor is the size of the NPs. Small NPs (less than $20 \mathrm{~nm}$ ) own superparamagnetic properties, which can be used to increase contrast in MRI (Jarockyte et al. 2016). The superparamagnetic iron oxide nanoparticle (SPION) consists usually of an iron oxide core (for MRI contrast, hyperthermia therapy, and controlled drug release) coated by a polymer coating (which prevents NP aggregation, extends their half-life time, regulates the drug release, and enables conjugation with other biomolecules). 
In addition, several biomolecules are conjugated to the polymer coat such as chemotherapeutical agents (one or more substances), photofunctional moiety (fluorescent dye or photosensitizing element), biotherapeutics (DNA/RNA/ proteins for gene therapy or hormone compensations), targeting agent (antibodies targeting the cancer cells or intracellular organelles), and functional moiety (cross physiological barriers and cell membranes) (Revia and Zhang 2016).

The ability of accumulated iron oxide NPs in the cancer cells to convert the electromagnetic energy into thermal energy when exposed to external magnetic field is exploited. The therapeutic hyperthermia concept utilizes the generated heat to kill cancer cells or make them more susceptible to be eliminated by other anti-cancer agents. The intensity and duration of the generated heat by these nano-heaters can be controlled by regulating the intensity and duration of oscillation of the external magnetic field. However, the used NPs must be very small in size to gain supermagnetic behavior, i.e., they lose their magnetic power directly when the magnetic field is removed. This is a momentous feature to prevent their aggregation which may lead to blockage of blood capillaries and prevents their passage through the endothelium of the capillary walls to the cancer cells. By the application of the external electromagnetic field, heat can be generated with a temperature up to $42{ }^{\circ} \mathrm{C}$ which is usually enough for the denaturation of intracellular proteins and the inactivation of the enzymatic system inside the cancer cells (Ishizaki et al. 2016; Rivas et al. 2012; Walter and Carraretto 2016; Xu and Sun 2013). The released heat leads also to the destruction of the extracellular matrix which facilitates the drug penetration in the tumor cells. Lab animal experiments revealed the death of $53 \%$ of the colo-rectal cancer cells within $6 \mathrm{~min}$, while only $5 \%$ of the adjacent healthy tissues were affected (Revia and Zhang 2016). Taking into consideration the safety aspects and biocompatibility, the use of iron oxide-based core shells or in its colloidal form draws greater attention in cancer therapy than other nanoformulations (Banobre-Lopez et al. 2013).

The magnetic hyperthermia is also used as a smart ondemand drug dosing tool where the drugs are coated with thermolabile polymers which shrink by heat exposure leading to intelligent/controlled drug release every time it is heated through the external magnetic field (Banobre-Lopez et al. 2013; Piñeiro et al. 2015). Additionally, SPION enables simultaneous monitoring of drug delivery/distribution and tissue response, so that the therapeutic interference can be adjusted according to the needs and results (Revia and Zhang 2016).

Other cancer therapeutic concepts were also developed and displayed promising results. Among them, the photon activation therapy (PAT) is based on the direction of NP to the tumor cells before being activated by synchrotron radiation (or Xray) to start a cascade of reaction. The released energy will result in the formation of ionization tracks which destroy the tumor cells. In laboratory animal experiments, tumor regression was reported within 2-4 weeks after treatment (Elgqvist 2017).

Photochemotherapy or photodynamic therapeutical assay (PDT) was also developed. The assay depends on the use of chemical substance (called photo-sensitizer), which produces nascent reactive oxygen upon radiation with certain light wavelengths. Tumor necrosis follows as a result of the cytotoxic effect of the nascent oxygen. The use of the PDT showed auspicious results especially in the treatment of skin cancer (Gupta et al. 2016).

A third photon dependent approach is the photothermal therapy (PTT)/plasmonic PTT (PPTT). The exposure of gold NP to laser light converts the light to heat energy. The use of gold NP in photothermal cancer therapy can be substituted by iron oxide NPs which have a gold shell (hybrid NPs). The delivered heat kills the tumor cells and causes necrosis of the affected area without releasing oxygen in opposite to the PDT (Revia and Zhang 2016; Turcheniuk et al. 2016).

Beside these physical approaches for cancer treatment, immunological assays were also investigated. Different NPs are used for cancer immunotherapy which vary in their chemical structure and mode of action. While some preparations can directly activate the immune cells, or increase the immunogenicity of the tumor cells, other preparations can prolong the drug effect in the cancer microenvironment (Dobrovolskaia et al. 2016). The concept of cancer immunotherapy is to modify T-lymphocytes in vitro to be able to express an artificial signaling molecule (CAR). The modified lymphocytes gain the ability to recognize and attack surface antigens expressed by cancer cells only. The T cells are usually suppressed by the programmed death-ligand 1 (PD-L1) protein produced by many cancer types (e.g., prostate cancer). The protein suppresses the $\mathrm{T}$ cell migration, proliferation, and their ability to produce cytotoxic mediators in order to kill the cancer cells. The development of anti-PD-L1 protein enables the recognition of the cancer cells by the immune system and the mobilization of immune cells to cancer microenvironment (Herbst et al. 2014).

A major obstacle in cancer therapy is the presence of certain types of cancer which are very aggressive and can resist conventional treatment. One of the futural aspects in cancer therapy is the use of the gene therapy to shut off major cancer regulatory genes. The main challenge in the approach is the development of synthetic RNA fragments with short half-lives in the circulation (Brede and Labhasetwar 2013). Cancer gene therapy became one of the most promising concepts in tumor treatment protocols. The treatment success degree depends on the type of the cancer and its stage, the immune status of the patient, and whether the gene therapy is given alone or coupled with chemotherapy/radiation. The delivery of the genetic materials into the nucleus is not an easy process. It could be achieved by the aid of synthetic viruses as carriers of the 
genetic material. However, the application of NPs allows their parallel use as therapeutic and imaging agents simultaneously (Kozielski et al. 2016). Gene therapy of cancer usually targets either the tumor-suppressor genes or the oncogenes such as short hairpin RNA (shRNA) or the small interfering RNA (siRNA) (Sridharan and Gogtay 2016). siRNA is a class of double-stranded RNA molecules which is responsible for mRNA cleavage. They are used in gene silencing in cancer cells (Braicu et al. 2015; Jurj et al. 2017). NPs used in cancer gene therapy are usually coated with a cationic polymer to bind easily to the negatively charged DNA fragments on their surface (Revia and Zhang 2016). Hepatocellular carcinoma (HCC) is responsible for one million deaths every year, being the second leading cause of cancer-related mortalities worldwide. The use of siRNA to silence HCC gene expression is a promising tool to treat $\mathrm{HCC}$ due to its high specificity, safety, and efficiency. However, the rapid degradation of the siRNA and its poor cellular uptake make the efficiency of its systemic delivery very low. The siRNA has a high molecular weight and negative charge which complicates the intracellular transport to the endosomes. This obstacle can be overcome by the use of the nanovector (NP-siRNA-GPC3 Ab). The vector was developed by (Wang et al. 2016) and consists of an iron oxide core covered by chitosan PEG-grafted PEI copolymer. The NP is functionalized by siRNA. The complex is then conjugated to a monoclonal antibody specific for the glypican-3 (GPC3) receptor (Wang et al. 2016). Successful field trials were carried out using NPs conjugated to siRNA where the cyclic nanopeptide siRNA conjugated to the outer surface of lipid-based carriers was able to target and penetrate the target cancer cells. Following penetration, the peptides are enzymatically processed by proteolytic enzymes of the cells to release their payload in the cancer cells (Parvanian et al. 2017). Advanced cationic polymer-decorated SPIO NPs containing disulfide bonds in their structures were also proven to be highly effective and safe for the delivery of nucleic acid or siRNA into cancer cells ( $\mathrm{Li}$ et al. 2014).

Recent works show that coupling the chemotherapeutic agents to NP enhances their biocompatibility, provides specificity and selectivity to target tumor cells alone. In addition, the NPs protect the drugs from degradation. All these factors allow the administration of higher doses with fewer side effects. Among different investigated agents, the combination of SPIONs with temozolomide (TMZ) and chlorotoxin (CTX) increase their stability and specificity (Fang et al. 2015; Revia and Zhang 2016).

In biotherapeutics, in opposite to chemotherapy, the NPs are designed to deliver biological materials such as DNA, siRNA, peptides, or even proteins instead of chemotherapeutical drugs in the cancer cells. Providing tumor cells with healthy DNA to replace defective genes will result in the expression of normal proteins instead of atypical ones. The same goal may be accomplished by supplying the tumor cells with siRNA or the required proteins. The delivered proteins may enhance important cellular functions as cell apoptosis. The use of NPs to carry the biological materials protects them from being attacked by the immune system (Revia and Zhang 2016; Xing et al. 2014).

Radiotherapy is one of the oldest concepts of cancer treatment. It depends on radiating the lesions with high doses of radiation which damage the DNA of the cancer cells and neighbor healthy tissues. At the time, radiolabeled NPs are commonly used in cancer diagnosis and therapy. However, serious concern about possible side effects may occur if the radionuclides escape to healthy tissues. This may occur if the binding between the radionuclides and the NPs is not stable enough. Other concerns about possible contamination during manufacturing and other health hazards for the workers were also taken into consideration (Wu et al. 2017).

Some NPs own more potency for cancer than others such as gold NPs, liposomes, hybrid systems, dendrimers, and CNTs (Jurj et al. 2017) Nevertheless, the use of a cocktail of a mixture of many anti-cancer nanodrugs with different therapeutic concepts was shown to have greater therapeutic potency (Casals et al. 2017), e.g., the use of a cocktail of NPs could reverse the resistance of breast cancer to tamoxifen and prevents further growth of the tumor by preventing the estrogen to bind estrogen receptors (Cho et al. 2013). Other cocktails were also designed to increase the intracellular drug uptake and accumulation in the MDR cancer cells (Gao et al. 2017).

\section{Gene and stem cell therapy and regenerative medicine}

In gene therapy, the function of a defective gene is to be replaced by a functioning variant of the gene. An integrating or non-integrating exogenous genetic material is introduced into the cell with the aid of special vectors in order to influence gene expression in living organisms. Successful gene therapy can be achieved via one of two approaches, either delivering DNA to the cells suffering from genetic disorders or inherited diseases to replace defective gene or delivery of therapeutic nucleic acids to silence genes as in cancer therapy. For gene therapy, anti-sense single-stranded 8-50-bp oligonucleotide complementary to the target mRNA is used. They are modified to resist inactivation by nuclease enzymes. Similarly, the DNA aptamers can be used in gene therapy. These are 56120-bp single-stranded nucleic acid which binds protein encoding genes (Riley and Vermerris 2017; Sridharan and Gogtay 2016).

For a long time, the viral vectors were used in gene therapy to deliver genetic materials due to their high transfection efficiency (Kamel and El-Sayed 2019). This application has many concerns about their biosafety (oncogenicity, mutagenesis, and immunogenicity). The use of NPs as carriers for genetic material is safer and allows loading with larger 
amounts of DNA. However, some challenges still face the design of carrier NPs such as their penetration through the cell membranes and to escape endosomal degradation following their endocytosis. After endocytosis, the substances are delivered to the endosomes are identified, sent to the lysosomes for degradation, or to the Golgi apparatus for processing, or removed back via plasma membrane (Gu et al. 2001; Luzio et al. 2007), So that, for successful gene therapy, the transported genes must pass through the cell membrane, lysosomes, and vector capsule without being degraded. For this purpose, biodegradable cationic polymers capable of endosomal escape are used (Li et al. 2014; Manuja et al. 2012).

Intelligent NPs are used in gene therapy due to their ability to protect the loaded DNA or RNA from degradation during their journey and due to their capability to enter the cell nucleus to deliver the genetic materials at their target. The intelligent NPs have a special coat which breaks down when exposed to certain environmental conditions present at their target organelle (Revia and Zhang 2016), e.g., iron oxide-cored NPs coated by the cationic polymer polyethyleneimine (PEI). The positively charged polymers bind easily to the negatively charged DNA. The bioreducible cationic polymers are biodegraded inside the cells. The PEI contains a disulfide bond which is usually stable outside the cells. When the NP enters the cell, the bond is cleaved by the glutathione leading to the release of the genetic materials. An additional advantage of the use of biodegradable polymers is their low cytotoxicity (Son et al. 2011). Chitosan can be also used as a coating material for particle stability, biocompatibility, and biodegradability (Cheng et al. 2011). Gold NPs are preferred for use in gene therapy. The flexible surfaces enable better functionalization and allow the DNA to be directly complexed to the NPs (Sattler 2010).

Another tool for gene therapy is based on the use of cellpenetrating peptides. They are cationic and/or amphipathic peptides. They are maximum 30 A.A. and can penetrate the cells with their load of RNA/DNA by energy depending endocytosis (Lehto et al. 2016). However, they have many disadvantages such as their low encapsulation efficiency, slow endosomal escape, and poor stability when stored. They can be loaded with small RNA molecules (miRNA/siRNA) for the induction of gene silencing in cancer cells, as well as they can be loaded with monoclonal antibodies to target antigens/cell receptors (Jurj et al. 2017). miRNA are short (20-25 nucleotides) single-stranded noncoding RNAs. They are responsible for the regulation of the post-transcriptional gene expression being implicated in cell differentiation, proliferation, metabolism, tumorigenesis, and apoptosis. When conjugated to NPs, they can restore normal gene expression or inhibit translation of mRNA in target cells (Braicu et al. 2015; Jurj et al. 2017).

Stem cells are characterized by their restricted cellular uptake; therefore, they are arduous to manipulate using conventional non-viral vectors (Lakshmipathy et al. 2004). This obstacle can be overwhelmed by the use of peptideconjugated gold NPs to deliver the DNA to target stem cells (Peng et al. 2014). Many investigators studied the application of fluorescent nano-diamond in stem cell therapy and regenerative medicine. The fluorescent nano-diamonds are natural biocompatible nontoxic NPs (Wu et al. 2017).

\section{Tissue engineering and prosthetic orthopedic implants}

Nanomaterials can be engineered to replace diseased and damaged tissues. Nanomaterials support tissue repair and regeneration, increase the durability and lifespan of implants, and minimize possible side effects and risk of rejection. With the aid of the nanomaterials, it is expected that artificial organs can be manufactured to replace damaged organs or tissues in the near future (Manuja et al. 2012).

Bone grafting is required in many cases when the condition exceeds the internal potency for bone regeneration such as in large scale injuries, infections, or tumors. Beside the bone tissue, it must be taken into consideration that bones contain in addition various proteins, such as collagen, fibronectin, and laminin, extracellular matrix, and bone supporting tissues, e.g., ligaments and tendons, which may need also large scale repair/regeneration. At present, many synthetic matrixes are in use; however, they suffer from implant material failure after 10-15 years due to various reasons (Zhang and Webster 2009). The used material should also be slowly bioabsorbable to allow its gradual replacement with bone materials (Yi et al. 2016). Nanomaterials were developed to offer biocompatible and safe surfaces which mimic natural bone environment. The used materials should enhance the normal healing process and the adhesion/proliferation of osteoblasts should have enough mechanical strength, be porous to allow transport of oxygen/ nutrients, and in the same time should not toxic during implantation or later (Williams 2008). Multiwalled carbon nanotubes were developed to be applied for the repair of tendons and ligaments. The extracellular materials are replaced by hydrogel which enhances cell proliferation and vascularization (Sheikh et al. 2015). It is also preferred to coat the metallic implants with nanomaterials to minimize debris degeneration and advocating an inflammatory response which prolongs the life expectation of the graft. The nanomaterial can also be loaded with antimicrobial drugs to prevent post-operative infections (Venkatesan and Kim 2014; Yi et al. 2016).

\section{Immunization and vaccine production}

For vaccine production, the NPs provide many advantages compared with conventional vaccines and adjuvants. They improve the solubility of hydrophobic antigens, have less post-vaccinal side effects, afford a controlled sustainable 
release of the antigens, target directly the lymph nodes or reticuloendothelial tissues, and require smaller volumes and less number of doses (Dobrovolskaia et al. 2016). They can also be constructed to be more immunogenic with adjuvant properties, carry antigens of different pathogens at the same time, and are safer to use. They are, in contrast to conventional ones, not susceptible for degradation and are effective for immunization against protected micro-organisms such as microbial spores. Successful trials to develop spore-based antiBacillus subtilis and anti-clostridium tetani vaccines were published (Manuja et al. 2012; Nordly et al. 2009). Supplying the NPs with pathogen-specific antigens advocates immune response. Some non-degradable biocompatible nanocarriers have additional adjuvant effect such as the natural zeolite clinoptilolite (Dobrovolskaia et al. 2016). Through mimicking the microbial antigens, NPs can stimulate active immune response. They may be also designed to upregulate definite genes responsible for the production of co-stimulatory substances in the antigen presenting cells. Through this mode of action, the immune response can be strengthened and prolonged. Alternatively, the NPs may be designed to release the loaded antigens gradually, which in turn increase the protection time (Mohantya et al. 2014; Nordly et al. 2009).

Nanovaccines and nano-adjuvants can be given separately or combined in one shot to reduce the number of required doses. Nanovaccines can be administrated via different routes which offer more flexibility making them ideal for veterinary applications specially if a large number of birds or animals are to be vaccinated (El-Sayed and Kamel 2018; Kamel et al. 2019). Topical application of nanovaccines on mucus membranes reduces the pain/fear of injections and provides better target mucosal immunity in the target systems (e.g., intranasal MM to protect the respiratory system) (Underwood and Van Eps 2012).

Nanotechnology allows the manipulation of the NPs to specifically target the immune system (for vaccine preparation) or to avoid its stimulation (for other medical applications) (Dobrovolskaia et al. 2016). There are several factors which determine the nature of immune response and the degree of phagocytic clearance of the NP, such as the NP size. It was found that NPs (20-200 nm) provoke strong CD4+ and CD8+ T cell responses while the same NPs but with larger size $(>500 \mathrm{~nm}$ ) have more potential to induce interferon (IFN)- $\gamma$ and antibody responses. Meanwhile, the larger counterpart ( $1200 \mathrm{~nm}$ ) NPs induced tumor necrosis factor (TNF)- $\alpha$. This difference is attributed to the preference of phagocytic cells to clear certain size ranges, and NPs ranging from100-200 nm are usually cleared by the Kupffer cells in the liver, while larger NPs are cleared by the splenic macrophages. Other factors which can also affect the degree of immune response include the materials used in the manufacturing of the NP, surface charge, and type of coating (Gbadamosi et al. 2002; Rettig et al. 2010; Xiang et al. 2010). After intradermal administration of small NPs $(<100 \mathrm{~nm})$, they directly target LN- resident dendritic cells (DCs), B cells, and macrophages. They are carried to the LNs via lymph drainage or by cell-associated migration. Meanwhile, the transportation of larger NPs is limited to their uptake by tissue-resident antigen presenting cells (Manolova et al. 2008). NPs are also employed to suppress undesired immune response and inflammation, e.g., the inhalation of carbon nanotubes results in systemic suppression of humoral immune response while allergic reactions (type I hypersensitivity) can be inhibited by the administration of fullerene derivative polyhydroxy C60 (Mitchell et al. 2009; Ryan et al. 2007).

Alternative to NPs, non-infectious virus-like particles (VLPs) can be used as carriers for the pathogen antigens or synthetic nucleotides (Kamel and El-Sayed 2019; Troncarelli et al. 2013). The VLPs are multiprotein structures similar to the viral structural proteins without containing viral genetic materials. They are safe and can be loaded with other antigens from other pathogens in parallel. Trials to produce VLP vaccines against $\mathrm{HBV}$ and human papillomavirus delivered successful results.

\section{Executive summary and conclusion}

The newly developed nanotechnological assays lead to a medical revolution in the diagnosis and treatment of both infectious and non-infectious diseases as drug-resistant pathogens, intracellular pathogens, different cancer types, and even many chronic non-infectious diseases. Various nanomaterials depending on the physical and chemical properties of their surface have been applied for varieties of biomedical applications such as drug delivery systems, optical imaging, bioimaging, biosensing, and bone grafting. Nanotechnology promoted in parallel advances in gene therapy and vaccine preparation. NPs have many merits in immunization and vaccine production as for instance they improve hydrophobic antigen solubility, have fewer side effects following vaccination, sustainable and controlled release of the antigens, target directly to the tissues, and need smaller volumes and less number of doses and more. NPs can also provoke active immune response carrying multiple antigens from several pathogens without degradation. The nature of these immune responses and the degree of phagocytic clearance of the NPs are affected by size of NPs, the materials used in manufacturing NPs, type of coating, and surface charge.

\section{Compliance with ethical standards}

Conflict of interest The authors declare that they have no conflict of interest.

Consent for Publication Not applicable (This article does not contain any studies with human participants or animals performed by any of the authors). 


\section{References}

Ahn-Yoon S, DeCory TR, Baeumner AJ, Durst RA (2003) Gangliosideliposome immunoassay for the ultrasensitive detection of cholera toxin. Anal Chem 75:2256-2261

Ahn-Yoon S, DeCory TR, Durst RA (2004) Ganglioside-liposome immunoassay for the detection of botulinum toxin. Anal Bioanal Chem 378:68-75

Aparicio MG, Iglesias A, Martinez E, Escolar P, Salinas J (2013) Initial outcomes in lung cancer treatment with VMAT. Rep Pract Oncol Radiother 2:9-13 S264

Awate S, Babiuk LAB, Mutwiri G (2013) Mechanisms of action of adjuvants. Front Immunol 4:114

Banobre-Lopez M, Teijeiro A, Rivas J (2013) Magnetic nanoparticlebased hyperthermia for cancer treatment. Rep Pract Oncol Radiother 18:397-400

Beyth N, Houri-Haddad Y, Domb A, Khan W, Hazan R (2015) Alternative antimicrobial approach: nano-antimicrobial materials. Evid Based Compl Alternat Med 2015:16

Blanco E, Hsiao A, Mann AP, Landry MG, Meric-Bernstam F, Ferrari M (2011) Nanomedicine in cancer therapy: innovative trends and prospects. Cancer Sci 102:1247-1252

Brahim S, Narinesingh D, Guiseppi-Elie A (2002) Bio-smart hydrogels: co-joined molecular recognition and signal transduction in biosensor fabrication and drug delivery. Biosens Bioelectron 17:973-981

Braicu C, Cojocneanu-Petric R, Chira S, Truta A, Floares A, Petrut B, Achimas-Cadariu P, Berindan-Neagoe I (2015) Clinical and pathological implications of miRNA in bladder cancer. Int J Nanomed 10: 791

Brede C, Labhasetwar V (2013) Applications of nanoparticles in the detection and treatment of kidney diseases. Adv Chronic Kidney Dis 20:454-465

Cao Y, Wang B, Lou D, Wang Y, Hao S, Zhang L (2011) Nanoscale delivery systems for multiple drug combinations in cancer. Future Oncol 7:1347-1357

Casals E, Gusta MF, Cobaleda-Siles M, Garcia-Sanz A, Puntes VF (2017) Cancer resistance to treatment and antiresistance tools offered by multimodal multifunctional nanoparticles. Cancer Nanotechnol 8:7

Ceña V, Játiva P (2018) Nanoparticle crossing of blood-brain barrier: a road to new therapeutic approaches to central nervous system diseases. 13:1513-1516

Chakravarthi PV, Balaji SN (2010): Applications of nanotechnology in veterinary medicine. Vet World 3

Chapman S, Dobrovolskaia M, Farahani K, Goodwin A, Joshi A, Lee H, Meade T, Pomper M, Ptak K, Rao J (2013) Nanoparticles for cancer imaging: The good, the bad, and the promise. Nano Today 8:454 460

Cheng R, Feng F, Meng F, Deng C, Feijen J, Zhong Z (2011) Glutathioneresponsive nano-vehicles as a promising platform for targeted intracellular drug and gene delivery. J Control Release 152:2-12

Cho SK, Pedram A, Levin ER, Kwon YJ (2013) Acid-degradable coreshell nanoparticles for reversed tamoxifen-resistance in breast cancer by silencing manganese superoxide dismutase (MnSOD). Biomaterials 34:10228-10237

Chu M (2017): Semiconductor quantum dots and rods for in vivo imaging and cancer phototherapy. World Sci

Dastjerdi R, Montazer M (2010) A review on the application of inorganic nano-structured materials in the modification of textiles: focus on anti-microbial properties. Colloids Surf B: Biointerfaces 79:5-18

Dear JW, Kobayashi H, Brechbiel MW, Star RA (2006) Imaging acute renal failure with polyamine dendrimer-based MRI contrast agents. Nephron Clin Pract 103:c45-c49
Dobrovolskaia MA, Shurin M, Shvedova AA (2016) Current understanding of interactions between nanoparticles and the immune system. Toxicol Appl Pharmacol 299:78-89

Duan L, Wang Y, Li SS-C, Wan Z, Zhai J (2005) Rapid and simultaneous detection of human hepatitis $\mathrm{B}$ virus and hepatitis $\mathrm{C}$ virus antibodies based on a protein chip assay using nano-gold immunological amplification and silver staining method. BMC Infect Dis 5:53

Ebrahim S, Reda M, Hussien A, Zayed D (2015) CdTe quantum dots as a novel biosensor for Serratia marcescens and lipopolysaccharide. Spectrochim Acta A Mol Biomol Spectrosc 150:212-219

El-Sayed A (2018) Advances in rabies prophylaxis and treatment with emphasis on immunoresponse mechanisms. Int J Vet Sci Med 6(1): $8-15$

El-Sayed A, Kamel M (2018) Advanced applications of nanotechnology in veterinary medicine. Environmental Science and Pollution Research pp 1-14

Elgqvist J (2017) Nanoparticles as theranostic vehicles in experimental and clinical applications - focus on prostate and breast cancer. Int J Mol Sci 18:1102

Fang C, Wang K, Stephen ZR, Mu Q, Kievit FM, Chiu DT, Press OW, Zhang M (2015) Temozolomide nanoparticles for targeted glioblastoma therapy. ACS Appl Mater Interfaces 7:6674-6682

Fortin J-P, Gazeau F, Wilhelm C (2008) Intracellular heating of living cells through Néel relaxation of magnetic nanoparticles. Eur Biophys J 37:223-228

Freitas RA Jr (2005) Microbivores: artificial mechanical phagocytes using digest and discharge protocol. J Evol Technol 14:1-52

Freitas RA (2005) Current status of nanomedicine and medical nanorobotics. J Comput Theor Nanosci 2:1-25

Ganesan P, Ko H-M, Kim I-S, Choi D-K (2015) Recent trends in the development of nanophytobioactive compounds and delivery systems for their possible role in reducing oxidative stress in Parkinson's disease models. Int J Nanomed 10:6757

Gao W, Ye G, Duan X, Yang X, Yang VC (2017) Transferrin receptortargeted $\mathrm{pH}$-sensitive micellar system for diminution of drug resistance and targetable delivery in multidrug-resistant breast cancer. Int J Nanomed 12:1047-1064

Gbadamosi J, Hunter A, Moghimi SM (2002) PEGylation of microspheres generates a heterogeneous population of particles with differential surface characteristics and biological performance. FEBS Lett 532:338-344

Goluch ED, Nam J-M, Georganopoulou DG, Chiesl TN, Shaikh KA, Ryu KS, Barron AE, Mirkin CA, Liu C (2006) A bio-barcode assay for on-chip attomolar-sensitivity protein detection. Lab Chip 6:12931299

Gu F, Crump C, Thomas G (2001) Trans-Golgi network sorting. Cell Mol Life Sci CMLS 58:1067-1084

Gupta A, Landis RF, Rotello VM (2016) Nanoparticle-based antimicrobials: surface functionality is critical. F1000Research 5:F1000 Faculty Rev-364

Hajipour MJ, Fromm KM, Ashkarran AA, de Aberasturi DJ, de Larramendi IR, Rojo T, Serpooshan V, Parak WJ, Mahmoudi M (2012) Antibacterial properties of nanoparticles. Trends Biotechnol 30:499-511

Hakemi-Vala M, Rafati H, Aliahmadi A, Ardalan A (2017): Nanoemulsions: a novel antimicrobial delivery system, nano-and microscale drug delivery systems. Elsevier, pp. 245-266

Hameed ASH, Karthikeyan C, Ahamed AP, Thajuddin N, Alharbi NS, Alharbi SA, Ravi G (2016) In vitro antibacterial activity of $\mathrm{ZnO}$ and $\mathrm{Nd}$ doped $\mathrm{ZnO}$ nanoparticles against ESBL producing Escherichia coli and Klebsiella pneumoniae. Sci Rep 6:24312

Herbst RS, Soria J-C, Kowanetz M, Fine GD, Hamid O, Gordon MS, Sosman JA, McDermott DF, Powderly JD, Gettinger SN (2014) Predictive correlates of response to the anti-PD-L1 antibody MPDL3280A in cancer patients. Nature 515:563-567 
Hirsch LR, Stafford RJ, Bankson J, Sershen SR, Rivera B, Price R, Hazle JD, Halas NJ, West JL (2003) Nanoshell-mediated near-infrared thermal therapy of tumors under magnetic resonance guidance. Proc Natl Acad Sci 100:13549-13554

Ishizaki T, Yatsugi K, Akedo K (2016) Effect of particle size on the magnetic properties of $\mathrm{Ni}$ nanoparticles synthesized with trioctylphosphine as the capping agent. Nanomaterials 6:172

Jarockyte G, Daugelaite E, Stasys M, Statkute U, Poderys V, Tseng TC, Hsu SH, Karabanovas V, Rotomskis R (2016) Accumulation and toxicity of superparamagnetic iron oxide nanoparticles in cells and experimental animals. Int J Mol Sci 17:1193

Jeevanandam J, Barhoum A, Chan YS, Dufresne A, Danquah MK (2018) Review on nanoparticles and nanostructured materials: history, sources, toxicity and regulations. Beilstein J Nanotechnol 9:10501074

Jurj A, Braicu C, Pop L-A, Tomuleasa C, Gherman CD, BerindanNeagoe I (2017) The new era of nanotechnology, an alternative to change cancer treatment. Drug Design Dev Ther 11:2871-2890

Kamel M, El-Sayed A (2019) Utilization of herpesviridae as recombinant viral vectors in vaccine development against animal pathogens. Virus Res 270:197648

Kamel M, El-Sayed A, Castañeda Vazquez HJ (2019) Foot-and-mouth disease vaccines: recent updates and future perspectives. Arch Virol 164:1501-1513

Klostranec JM, Xiang Q, Farcas GA, Lee JA, Rhee A, Lafferty EI, Perrault SD, Kain KC, Chan WC (2007) Convergence of quantum dot barcodes with microfluidics and signal processing for multiplexed high-throughput infectious disease diagnostics. Nano Lett 7:2812-2818

Kozielski KL, Rui Y, Green JJ (2016) Non-viral nucleic acid containing nanoparticles as cancer therapeutics. Expert opinion on drug delivery 13:1475-1487

Kreuter J (2007) Nanoparticles - a historical perspective. Int J Pharm $331: 1-10$

Krishnan SR, George SK (2014): Nanotherapeutics in cancer prevention, diagnosis and treatment, pharmacology and therapeutics

Lakshmipathy U, Pelacho B, Sudo K, Linehan JL, Coucouvanis E, Kaufman DS, Verfaillie CM (2004) Efficient transfection of embryonic and adult stem cells. Stem Cells 22:531-543

Lehto T, Ezzat K, Wood MJ, Andaloussi SE (2016) Peptides for nucleic acid delivery. Adv Drug Deliv Rev 106:172-182

Leoni L, Desai TA (2004) Micromachined biocapsules for cell-based sensing and delivery. Adv Drug Deliv Rev 56:211-229

Li D, Tang X, Pulli B, Lin C, Zhao P, Cheng J, Lv Z, Yuan X, Luo Q, Cai $H$ (2014) Theranostic nanoparticles based on bioreducible polyethylenimine-coated iron oxide for reduction-responsive gene delivery and magnetic resonance imaging. Int $\mathrm{J}$ Nanomedicine 9: 3347

Li F, Li Y, Feng J, Dong Y, Wang P, Chen L, Chen Z, Liu H, Wei Q (2017) Ultrasensitive amperometric immunosensor for PSA detection based on $\mathrm{Cu} 2 \mathrm{O} @ \mathrm{CeO} 2-\mathrm{Au}$ nanocomposites as integrated triple signal amplification strategy. Biosens Bioelectron 87:630-637

Livney YD, Assaraf YG (2013) Rationally designed nanovehicles to overcome cancer chemoresistance. Adv Drug Deliv Rev 65:17161730

Luzio JP, Pryor PR, Bright NA (2007) Lysosomes: fusion and function. Nat Rev Mol Cell Biol 8:622-632

Mahmoudi M, Serpooshan V (2012) Silver-coated engineered magnetic nanoparticles are promising for the success in the fight against antibacterial resistance threat. ACS Nano 6:2656-2664

Manolova V, Flace A, Bauer M, Schwarz K, Saudan P, Bachmann MF (2008) Nanoparticles target distinct dendritic cell populations according to their size. Eur J Immunol 38:1404-1413

Manuja A, Kumar B, Singh RK (2012) Nanotechnology developments: opportunities for animal health and production. Nanotechnol Dev $2: 4$
Martin TA, Ye L, Sanders AJ, Lane J, Jiang WG (2013): Cancer invasion and metastasis: molecular and cellular perspective.

Meena N, Sahni Y, Thakur D, Singh R (2018) Applications of nanotechnology in veterinary. Vet World 3(10):477-480

Minchin RF, Martin DJ (2010) Nanoparticles for molecular imaging-an overview. Endocrinology 151:474-481

Mitchell L, Lauer F, Burchiel S, McDonald J (2009) Mechanisms for how inhaled multiwalled carbon nanotubes suppress systemic immune function in mice. Nat Nanotechnol 4:451-456

Mohantya N, Palaib T, Prustyc B, Mohapatrad J (2014) An overview of nanomedicine in veterinary science. Vet Res 2:90-95

Monami G, Emiliozzi V, Bitto A, Lovat F, Xu S-Q, Goldoni S, Fassan M, Serrero G, Gomella LG, Baffa R (2009) Proepithelin regulates prostate cancer cell biology by promoting cell growth, migration, and anchorage-independent growth. Am J Pathol 174:1037-1047

Morilla MJ, Montanari J, Frank F, Malchiodi E, Corral R, Petray P, Romero EL (2005) Etanidazole in pH-sensitive liposomes: design, characterization and in vitro/in vivo anti-Trypanosoma cruzi activity. J Control Release 103:599-607

Nordly P, Madsen HB, Nielsen HM, Foged C (2009) Status and future prospects of lipid-based particulate delivery systems as vaccine adjuvants and their combination with immunostimulators. Expert Opin drug delivery 6:657-672

Parvanian S, Mostafavi SM, Aghashiri M (2017) Multifunctional nanoparticle developments in cancer diagnosis and treatment. Sensing and Bio-Sensing Research 13:81-87

Patil S, Kore K, Kumar P (2009) Nanotechnology and its applications in Veterinary and Animal Science. Vet World 2:475-477

Pedrosa P, Baptista PV (2015) Gold and silver nanoparticles for diagnostics of infection, Nanotechnology in diagnosis, treatment and prophylaxis of infectious diseases. Elsevier, pp. 1-18

Peng L-H, Niu J, Zhang C-Z, Yu W, Wu J-H, Shan Y-H, Wang X-R, Shen Y-Q, Mao Z-W, Liang W-Q (2014) TAT conjugated cationic noble metal nanoparticles for gene delivery to epidermal stem cells. Biomaterials 35:5605-5618

Piñeiro Y, Vargas Z, Rivas J, López-Quintela MA (2015) Iron oxide based nanoparticles for magnetic hyperthermia strategies in biological applications. Eur J Inorg Chem 2015:4495-4509

Probst CE, Zrazhevskiy P, Bagalkot V, Gao X (2013) Quantum dots as a platform for nanoparticle drug delivery vehicle design. Adv Drug Deliv Rev 65:703-718

Qasim M, Lim D-J, Park H, Na D (2014) Nanotechnology for diagnosis and treatment of infectious diseases. J Nanosci Nanotechnol 14: 7374-7387

Qin D, He X, Wang K, Zhao XJ, Tan W, Chen J (2007) Fluorescent nanoparticle-based indirect immunofluorescence microscopy for detection of Mycobacterium tuberculosis. Biomed Res Int 2007:89364

Rapoport N, Gao Z, Kennedy A (2007) Multifunctional nanoparticles for combining ultrasonic tumor imaging and targeted chemotherapy. J Natl Cancer Inst 99:1095-1106

Remião MH, Segatto NV, Pohlmann A, Guterres SS, Seixas FK, Collares $\mathrm{T}$ (2018) The potential of nanotechnology in medically assisted reproduction. Front Pharmacol 8:994

Rettig L, Haen SP, Bittermann AG, von Boehmer L, Curioni A, Krämer SD, Knuth A, Pascolo S (2010) Particle size and activation threshold: a new dimension of danger signalling. Blood 115:4533-4541 blood-2009-11-247817

Revia RA, Zhang M (2016) Magnetite nanoparticles for cancer diagnosis, treatment, and treatment monitoring: recent advances. Mater Today (Kidlington) 19:157-168

Riley MK, Vermerris W (2017) Recent advances in nanomaterials for gene delivery-a review. Nanomaterials 7:94

Rivas J, Bañobre-López M, Piñeiro-Redondo Y, Rivas B, López-Quintela M (2012) Magnetic particles and clusters through a crossdisciplinary approach. J Magn Magn Mater 324:3499-3502 
Rizzo LY, Theek B, Storm G, Kiessling F, Lammers T (2013) Recent progress in nanomedicine: therapeutic, diagnostic and theranostic applications. Curr Opin Biotechnol 24:1159-1166

Rodríguez-Burneo N, Busquets MA, Estelrich J (2017) Magnetic Nanoemulsions: Comparison between nanoemulsions formed by ultrasonication and by spontaneous emulsification. Nanomaterials 7:190

Ryan JJ, Bateman HR, Stover A, Gomez G, Norton SK, Zhao W, Schwartz LB, Lenk R, Kepley CL (2007) Fullerene nanomaterials inhibit the allergic response. J Immunol 179:665-672

Sattler KD (2010): Handbook of nanophysics: nanomedicine and nanorobotics. CRC Press

Seleem MN, Jain N, Pothayee N, Ranjan A, Riffle JS, Sriranganathan N (2009) Targeting Brucella melitensis with polymeric nanoparticles containing streptomycin and doxycycline. FEMS Microbiol Lett 294:24-31

Shangguan J, Li Y, He D, He X, Wang K, Zou Z, Shi H (2015) A combination of positive dielectrophoresis driven on-line enrichment and aptamer-fluorescent silica nanoparticle label for rapid and sensitive detection of Staphylococcus aureus. Analyst 140:4489-4497

Sharma A, Pandey R, Sharma S, Khuller G (2004) Chemotherapeutic efficacy of poly (DL-lactide-co-glycolide) nanoparticle encapsulated antitubercular drugs at sub-therapeutic dose against experimental tuberculosis. Int J Antimicrob Agents 24:599-604

Sheikh FA, Macossay J, Cantu T, Zhang X, Hassan MS, Salinas ME, Farhangi CS, Ahmad H, Kim H, Bowlin GL (2015) Imaging, spectroscopy, mechanical, alignment and biocompatibility studies of electrospun medical grade polyurethane (Carbothane ${ }^{\mathrm{TM}} 3575 \mathrm{~A}$ ) nanofibers and composite nanofibers containing multiwalled carbon nanotubes. J Mech Behav Biomed Mater 41:189-198

Son S, Namgung R, Kim J, Singha K, Kim WJ (2011) Bioreducible polymers for gene silencing and delivery. Acc Chem Res 45: $1100-1112$

Sridharan K, Gogtay NJ (2016) Therapeutic nucleic acids: current clinical status. Br J Clin Pharmacol 82:659-672

Swain PS, Rajendran D, Rao S, Dominic G (2015) Preparation and effects of nano mineral particle feeding in livestock: A review. Vet world 8: 888-891

Talukdar D, Sharma R, Sharma AK, Kumar R (2014) Drug resistance in tuberculosis: how to counter the menace? Curr Pharm Biotechnol 15:1158-1165

Tang F, Pang D-W, Chen Z, Shao J-B, Xiong L-H, Xiang Y-P, Xiong Y, Wu K, Ai H-W, Zhang H (2016) Visual and efficient immunosensor technique for advancing biomedical applications of quantum dots on salmonella detection and isolation. Nanoscale 8:4688-4698

Torres-Sangiao E, Holban AM, Gestal MC (2016) Advanced nanobiomaterials: vaccines, diagnosis and treatment of infectious diseases. Molecules 21:867

Troncarelli MZ, Brandão HM, Gern JC, Guimarães AS, Langoni H (2013) Nanotechnology and antimicrobials in veterinary medicine. FORMATEX, Badajoz

Turcheniuk K, Dumych T, Bilyy R, Turcheniuk V, Bouckaert J, Vovk V, Chopyak V, Zaitsev V, Mariot P, Prevarskaya N (2016) Plasmonic photothermal cancer therapy with gold nanorods/reduced graphene oxide core/shell nanocomposites. RSC Adv 6:1600-1610

Underwood C, Van Eps A (2012) Nanomedicine and veterinary science: the reality and the practicality. Vet J 193:12-23

Varkouhi AK, Scholte M, Storm G, Haisma HJ (2011) Endosomal escape pathways for delivery of biologicals. J Control Release 151:220 228

Venkatesan J, Kim S-K (2014) Nano-hydroxyapatite composite biomaterials for bone tissue engineering - a review. J Biomed Nanotechnol 10:3124-3140

Villaverde A (2011): Nanoparticles in translational science and medicine, 104. Academic Press

Vinogradov S, Wei X (2012) Cancer stem cells and drug resistance: the potential of nanomedicine. Nanomedicine 7:597-615

Walter EJ, Carraretto M (2016) The neurological and cognitive consequences of hyperthermia. Crit Care 20:199

Wang K, Kievit FM, Sham JG, Jeon M, Stephen ZR, Bakthavatsalam A, Park JO, Zhang M (2016) Iron-oxide-based nanovector for tumor targeted siRNA Delivery in an orthotopic hepatocellular carcinoma xenograft mouse model. Small 12:477-487

Wang Q-Y, Kang Y-J (2016) Bioprobes based on aptamer and silica fluorescent nanoparticles for bacteria salmonella typhimurium detection. Nanoscale Res Lett 11:150

Williams DF (2008) On the mechanisms of biocompatibility. Biomaterials 29:2941-2953

Wu T-J, Chiu H-Y, Yu J (2017): Nanotechnologies for early diagnosis, in situ disease monitoring, and prevention. Nanotechnologies in Preventive and Regenerative Medicine: An Emerging Big Picture, 1

Xiang SD, Selomulya C, Ho J, Apostolopoulos V, Plebanski M (2010) Delivery of DNA vaccines: an overview on the use of biodegradable polymeric and magnetic nanoparticles. Wiley Interdisciplinary Reviews: Nanomedicine and Nanobiotechnology 2:205-218

Xing R, Liu G, Zhu J, Hou Y, Chen X (2014) Functional magnetic nanoparticles for non-viral gene delivery and MR imaging. Pharm Res 31:1377-1389

Xu C, Sun S (2013) New forms of superparamagnetic nanoparticles for biomedical applications. Adv Drug Deliv Rev 65:732-743

Yi H, Rehman FU, Zhao C, Liu B, He N (2016) Recent advances in nano scaffolds for bone repair. Bone Res 4:16050

Zhang C-Y, Hu J (2010) Single quantum dot-based nanosensor for multiple DNA detection. Anal Chem 82:1921-1927

Zhang L, Webster TJ (2009) Nanotechnology and nanomaterials: promises for improved tissue regeneration. Nano Today 4:66-80

Zhao L, Seth A, Wibowo N, Zhao C-X, Mitter N, Yu C, Middelberg AP (2014) Nanoparticle vaccines. Vaccine 32:327-337

Zhou Y, Peng Z, Seven ES, Leblanc RM (2018) Crossing the blood-brain barrier with nanoparticles. J Control Release 270:290-303

Publisher's note Springer Nature remains neutral with regard to jurisdictional claims in published maps and institutional affiliations. 